\title{
Radiative Corrections to WW Scattering in the Standard Model
}

\author{
R. BOUAMRANE \\ Randall Laboratory of Physics \\ University of Michigan \\ Ann Arbor, MI 48109, USA
}

\section{Introduction}

The standard model of electroweak interactions has been consistent with all known experimental data to this date. However, there remain some important components of this model as yet unverified experimentally, namely the vector boson self-interactions and the Higgs sector. Both are needed to insure renormalizability without spoiling gauge invariance.

If the Higgs exists and is heavy but still light enough for perturbation theory to remain valid $\left(m_{\text {Higgs }}<1 \mathrm{TeV}\right)$, this calculation might provide a basic guideline for measuring deviations from the minimal Standard Model in future high energy WW scattering experiments. On the other hand, if the Higgs mass is much higher than $1 \mathrm{TeV}$, this calculation allows us to refine the unitarity bounds by taking into account the one-loop radiative corrections to the $\mathrm{J}=0$ partial-wave amplitude. (Of course, we do not know what higher order corrections will do.)

\section{The model}

The model we use is the minimal $S U(2) \times U(1)$ standard model. The Higgs sector is restricted to a single complex doublet. The free parameters of the model are:

$$
\begin{aligned}
g & \text { weak coupling constant } \\
s_{\theta}, c_{\theta} & \text { the sine and cosine of the weak mixing angle } \\
M & \text { mass of the charged vector boson } \\
m & \text { mass of the Higgs boson } \\
m_{t}^{\alpha}, m_{b}^{\alpha}, m_{e}^{\alpha} & \text { masses of quarks and leptons ( } \alpha \text { is the generation index). }
\end{aligned}
$$

We assume massless neutrinos.

All our calculations are done in the limit $m^{2} \gg s, t, u \gg M^{2}, m_{t}^{2}$ where $s, t$, and $u$ are the usual Mandelstam variables. Only longitudinally polarized vector bosons are 
considered. Furthermore, we consider the following three independent amplitudes:

$$
\begin{aligned}
\mathcal{A}_{a} & =\mathcal{A}\left(W_{L}^{+} W_{L}^{-} \rightarrow W_{L}^{+} W_{L}^{-}\right) \\
\mathcal{A}_{b} & =\mathcal{A}\left(W_{L}^{0} W_{L}^{0} \rightarrow W_{L}^{+} W_{L}^{-}\right) \\
\mathcal{A}_{c} & =\mathcal{A}\left(W_{L}^{0} W_{L}^{0} \rightarrow W_{L}^{0} W_{L}^{0}\right)
\end{aligned}
$$

\section{Tree level calculations}

The tree level amplitudes for $W_{L} W_{L}$ scattering have been extensively studied [1,2]. At energies very high with respect to the $W$ mass, we have the following:

$$
\begin{aligned}
& \mathcal{A}_{a}=\frac{g^{2} m^{2}}{4 M^{2}}\left(\frac{s}{-s+m^{2}}+\frac{t}{-t+m^{2}}\right) \\
& \mathcal{A}_{b}=\frac{g^{2} m^{2}}{4 M^{2}}\left(\frac{s}{-s+m^{2}}\right) \\
& \mathcal{A}_{c}=\frac{g^{2} m^{2}}{4 M^{2}}\left(\frac{s}{-s+m^{2}}+\frac{t}{-t+m^{2}}+\frac{u}{-u+m^{2}}\right)
\end{aligned}
$$

Taking $m^{2} \gg s, t, u$ we have for the $\mathrm{J}=0$ partial-wave amplitude:

$$
\begin{aligned}
& a_{a}^{0}=\frac{g^{2} s}{128 \pi M^{2}} \\
& a_{b}^{0}=\frac{g^{2} s}{64 \pi M^{2}} \\
& a_{c}^{0}=0
\end{aligned}
$$

The most important feature of the $\mathrm{J}=0$ partial-wave tree level amplitude for $W W$ scattering is the cancellation at high energy $\left(s \gg m^{2}, M^{2}\right)$ of the $s^{2}$ and $s$ terms from the $W$ exchange diagrams and similar terms from the four-W vertex and the Higgs exchange diagrams. This cancellation makes sure that tree level unitarity is respected at very high energies. This is true provided that the Higgs mass $m$ is not too large (less than $1 \mathrm{TeV}[1]$ ). In the case $m^{2} \gg s \gg M^{2}$, the $\mathrm{J}=0$ partial-amplitude stays linear as a function of $s$. (It is constant for $W^{0} W^{0} \rightarrow W^{0} W^{0}$ ). Tree level unitarity is violated at a critical energy $\sqrt{s^{*}} \simeq 1.7 \mathrm{TeV}$ [2]. This bound is obtained by considering the requirements of partial-wave unitarity on the two-channel system consisting of $W_{L}^{+} W_{L}^{-}$, and $\frac{1}{\sqrt{2}} W_{L}^{0} W_{L}^{0}$, with amplitudes given by (2). This is achieved by calculating the largest eigenvalue of the following $2 \times 2$ matrix:

$$
\left(\begin{array}{cc}
a_{a}^{0} & \frac{1}{\sqrt{2}} a_{b}^{0} \\
\frac{1}{\sqrt{2}} a_{b}^{0} & \frac{1}{2} a_{c}^{0}
\end{array}\right)
$$

\section{Renormalization}

All renormalization schemes involve some sort of redefinition of the parameters and the fields in the Lagrangian. For example [3]: 


$$
\begin{aligned}
g & \rightarrow g\left(1+\delta_{g}\right) \\
M & \rightarrow M\left(1+\delta_{M}\right) \\
c_{\theta} & \rightarrow c_{\theta}\left(1+\delta_{c_{\theta}}\right) \\
m & \rightarrow m\left(1+\delta_{m}\right) \\
W_{\mu}^{ \pm} & \rightarrow W_{\mu}^{ \pm}\left(1+\delta_{c}\right) \\
W_{\mu}^{0} & \rightarrow W_{\mu}^{0}\left(1+\delta_{0}\right)+\delta_{0 A} A_{\mu} \\
A_{\mu} & \rightarrow A_{\mu}\left(1+\delta_{A}\right)+\delta_{A 0} W_{\mu}^{0} \\
\phi^{ \pm} & \rightarrow \phi^{ \pm}\left(1+\delta_{H}\right) \\
\phi^{0} & \rightarrow \phi^{0}\left(1+\delta_{H}\right) \\
H & \rightarrow H\left(1+\delta_{H}\right)+\frac{M}{g} \delta_{t}
\end{aligned}
$$

Depending on the scheme prefered, the quantities $\delta_{g}, \delta_{M}$, etc. are chosen to compensate part of the one-loop corrections of the processes used as data input to fix $g, M$, etc. So, in principle, the one-loop corrections to these processes must be computed before any prediction on the four-W amplitude is made. This of course would be the case when doing an exact calculation. In the limit in which we are interested, namely $m^{2} \gg s, t, u \gg M^{2}$, the quantities $\delta_{g}, \delta_{M}$, etc. can be chosen as:

$$
\delta=a_{3} m^{2} \log \left(m^{2}\right)+a_{2} m^{2}+a_{1} \log \left(m^{2}\right)+a_{0},
$$

where the $a_{i}$ 's are constants to be determined.

The radiative corrections to the four- $\mathrm{W}$ amplitude are obtained by adding the contributions from all the diagrams shown in figs. 1 and 2. Diagrams with crosses involve the quantities $\delta_{g}$, etc.. Their associated Feynman rules were derived from the extra terms generated in the Lagrangian after the redefinitions (4) were made. (For some examples, see fig. 3.)

For the three processes considered in (1), the counter-terms' contributions as a function of the $\delta$ 's are:

$$
\begin{aligned}
& \mathcal{A}_{a}^{\text {counter }}=\frac{g^{2}}{32 \pi^{4} i M^{2} m^{2}}\left(\delta_{M}-\delta_{m}+\delta_{g}+2 \delta_{c}\right)\left(t^{2}+s^{2}\right) \\
& \mathcal{A}_{b}^{\text {counter }}=\frac{g^{2}}{64 \pi^{4} i M^{2} m^{2}}\left(\delta_{M}-\delta_{m}+\delta_{g}+\delta_{c}+\delta_{0}-\delta_{c_{\theta}}\right) s^{2} \\
& \mathcal{A}_{c}^{\text {counter }}=\frac{g^{2}}{32 \pi^{4} i M^{2} m^{2}}\left(\delta_{M}-\delta_{m}+\delta_{g}+2 \delta_{0}-2 \delta_{c_{\theta}}\right)\left(s^{2}+t^{2}+u^{2}\right)
\end{aligned}
$$

We notice that only the $m^{2}$ terms (if any) contained in the $\delta$ 's affect the one-loop order four-W amplitude. These terms were previously calculated by van der $\mathrm{Bij}$ and Veltman [3] and were shown to be unobservable at the one-loop level for processes not involving external Higgs lines. Thus, in the limit $m^{2} \gg s, t, u \gg M^{2}$, the one-loop order amplitude does not depend at all on the physical processes chosen to fix the free parameters $g, M$, etc. The quantities $\delta_{g}, \delta_{c}, \delta_{0}, \delta_{c_{\theta}}$ are free from $m^{2}$ dependence. The only relevant quantity is:

$$
\delta_{M}-\delta_{m}=-2 \pi^{2} i \frac{m^{2}}{M^{2}}\left[\frac{3}{4} \frac{1}{n-4}+\frac{3}{8} \log \left(m^{2}\right)-\frac{25}{32}+\frac{9 \pi}{32 \sqrt{3}}\right]
$$

The heart of the calculations is, of course, the evaluation of approximately 1000 loop diagrams using the algebraic manipulation program Schoonship [6]. We have 
kept the leading terms which are quadratic in $s, t, u$ with or without logarithms such as $\log \left(s / m^{2}\right)$, etc..

These diagrams can essentially be grouped into two sets. The first set consists of diagrams with no Higgs propagators inside the loop. We have found that the contributions from these diagrams essentially reduce to two-point functions and completely cancel in all the three processes at which we have looked. The second set consists of diagrams with Higgs propagators occurring in the loop. After appropriately expanding all the integrands, again, only two-point functions are left. (For calculational details, see (refs. [4].)

\section{Results}

The radiative corrections for the processes considered are:

$$
\begin{aligned}
\mathcal{A}_{a}^{1}= & -\frac{g^{4}}{16 \pi^{2} M^{4}}\left\{\frac{1}{18} t s+\left(\frac{5}{9}-\frac{9 \pi}{32 \sqrt{3}}\right)\left(t^{2}+s^{2}\right)\right. \\
& +\left(\frac{1}{96} t s+\frac{5}{96} s^{2}\right) \log \left(s / m^{2}\right) \\
& +\left(\frac{1}{96} t s+\frac{5}{96} t^{2}\right) \log \left(t / m^{2}\right) \\
& \left.+\frac{1}{32} u^{2} \log \left(u / m^{2}\right)\right\} \\
\mathcal{A}_{b}^{1}= & -\frac{g^{4}}{16 \pi^{2} M^{4}}\left\{\left(\frac{37}{72}-\frac{9 \pi}{32 \sqrt{3}}\right) s^{2}+\frac{1}{72} t^{2}+\frac{1}{72} u^{2}\right. \\
& +\frac{1}{32} s^{2} \log \left(s / m^{2}\right) \\
& +\left(\frac{1}{64} t^{2}+\frac{1}{192} u^{2}-\frac{1}{192} s^{2}\right) \log \left(t / m^{2}\right) \\
& \left.+\left(\frac{1}{64} u^{2}+\frac{1}{192} t^{2}-\frac{1}{192} s^{2}\right) \log \left(u / m^{2}\right)\right\} \\
\mathcal{A}_{c}^{1}= & -\frac{g^{4}}{16 \pi^{2} M^{4}}\left\{\left(\frac{13}{24}-\frac{9 \pi}{32 \sqrt{3}}\right)\left(s^{2}+t^{2}+u^{2}\right)\right. \\
& \left.+\frac{1}{16} s^{2} \log \left(s / m^{2}\right)+\frac{1}{16} t^{2} \log \left(t / m^{2}\right)+\frac{1}{16} u^{2} \log \left(u / m^{2}\right)\right\},
\end{aligned}
$$

The corresponding $J=0$ partial-wave amplitudes are:

$$
\begin{aligned}
& a_{a}=\frac{g^{2} s}{128 \pi^{2} M^{2}}\left[1+\frac{g^{2} s}{64 \pi^{2} M^{2}}\left(-\frac{20}{9} \log \left(s / m^{2}\right)-\frac{2441}{108}+\frac{12 \pi}{\sqrt{3}}\right)\right] \\
& a_{b}=\frac{g^{2} s}{64 \pi^{2} M^{2}}\left[1+\frac{g^{2} s}{64 \pi^{2} M^{2}}\left(-\frac{5}{9} \log \left(s / m^{2}\right)-\frac{905}{108}+\frac{9 \pi}{2 \sqrt{3}}\right)\right] \\
& a_{c}=\frac{g^{2} s}{128 \pi^{2} M^{2}}\left[0 .+\frac{g^{2} s}{64 \pi^{2} M^{2}}\left(-\frac{10}{3} \log \left(s / m^{2}\right)-\frac{256}{9}+\frac{15 \pi}{\sqrt{3}}\right)\right]
\end{aligned}
$$

These results are plotted in fig. 3. In fig. 4, the critical energy $\sqrt{s^{*}}$ obtained by coupling the channels involving $W_{L}^{+} W_{L}^{-}$and $\left(\frac{1}{\sqrt{2}}\right) W_{L}^{0} W_{L}^{0}$, with amplitudes given by (10), is plotted as a function of the Higgs mass (fig. 5). These results agree with [5]. 


\section{Conclusion}

The one-loop corrections are positive throughout the energy range of interest. $\mathrm{Nu}$ merically speaking, for a Higgs mass of $1 \mathrm{TeV}$, the radiative corrections to the tree amplitude ratio remain within $5 \%$ for energies below $500 \mathrm{GeV}$. For a heavier Higgs mass, the results we have presented should be taken only as an indication because perturbation theory is no longer valid. Nevertheless, for a very heavy Higgs (5-10 $T e V$ ), the critical energy at which the $\mathrm{J}=0$ partial-wave reaches unity is reduced by $(20-30 \%)$ when including the one-loop corrections.

\section{References}

[1] B. Lee, C. Quigg, and H. Thacker, Phys. Rev. D16, 1519 (1977)

[2] M. Chanowitz and M. Gaillard, Nucl. Phys. B261, 379 (1985)

[3] J. Van der Bij and M. Veltman, Nucl. Phys. B231, 205 (1984)

[4] M. Veltman and F.J. Yndurain, preprint UM-TH-89-04

R. Bouamrane, PhD thesis (1989), unpublished

[5] S. Dawson and S. Willenbrock, Phys. Rev. Let. 62, 1232 (1989)

[6] SCHOONSHIP, 68000 version Jan 11989. 

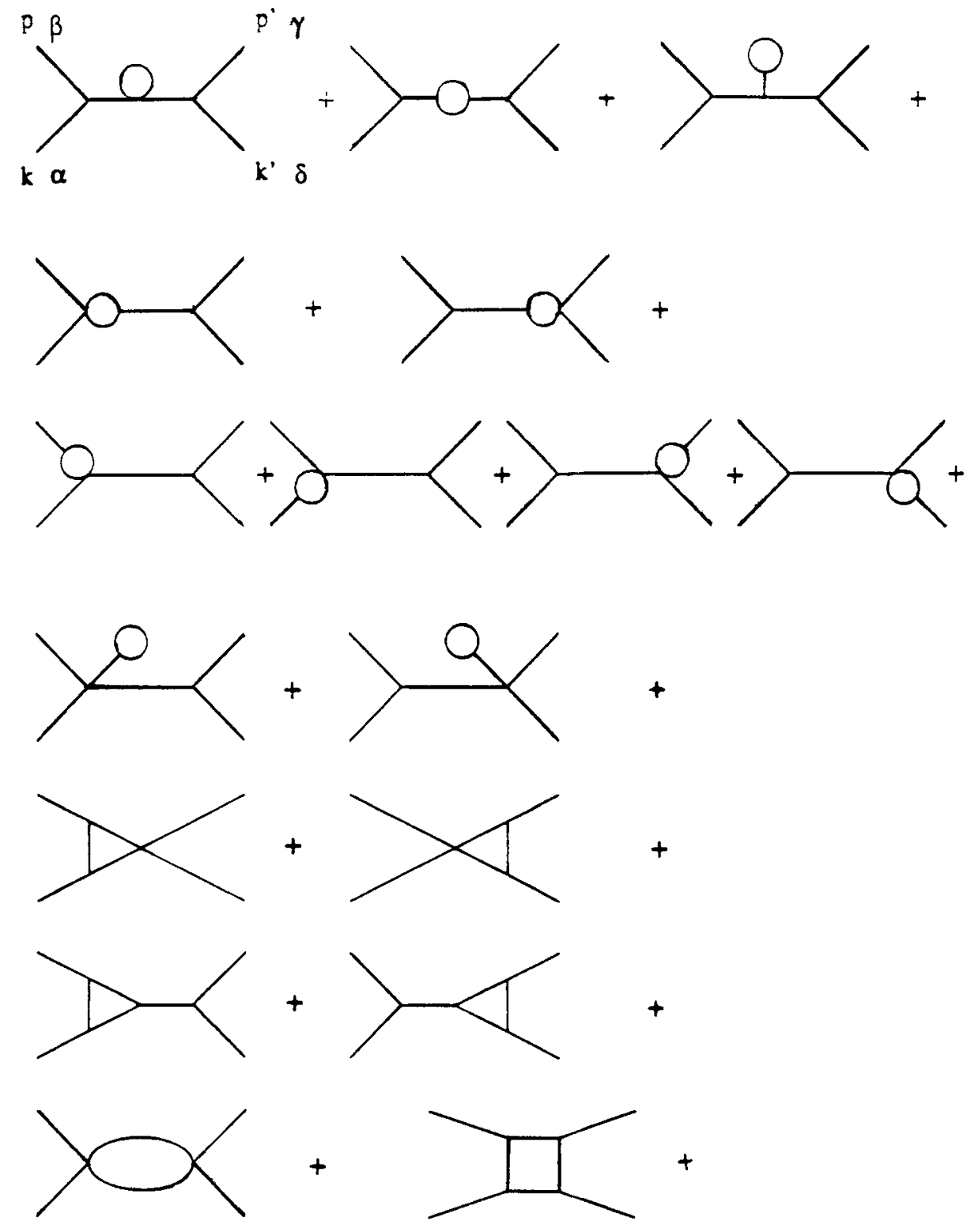

Figure 1: Topologies contributing to the one-loop corrections for $W W$ scattering 

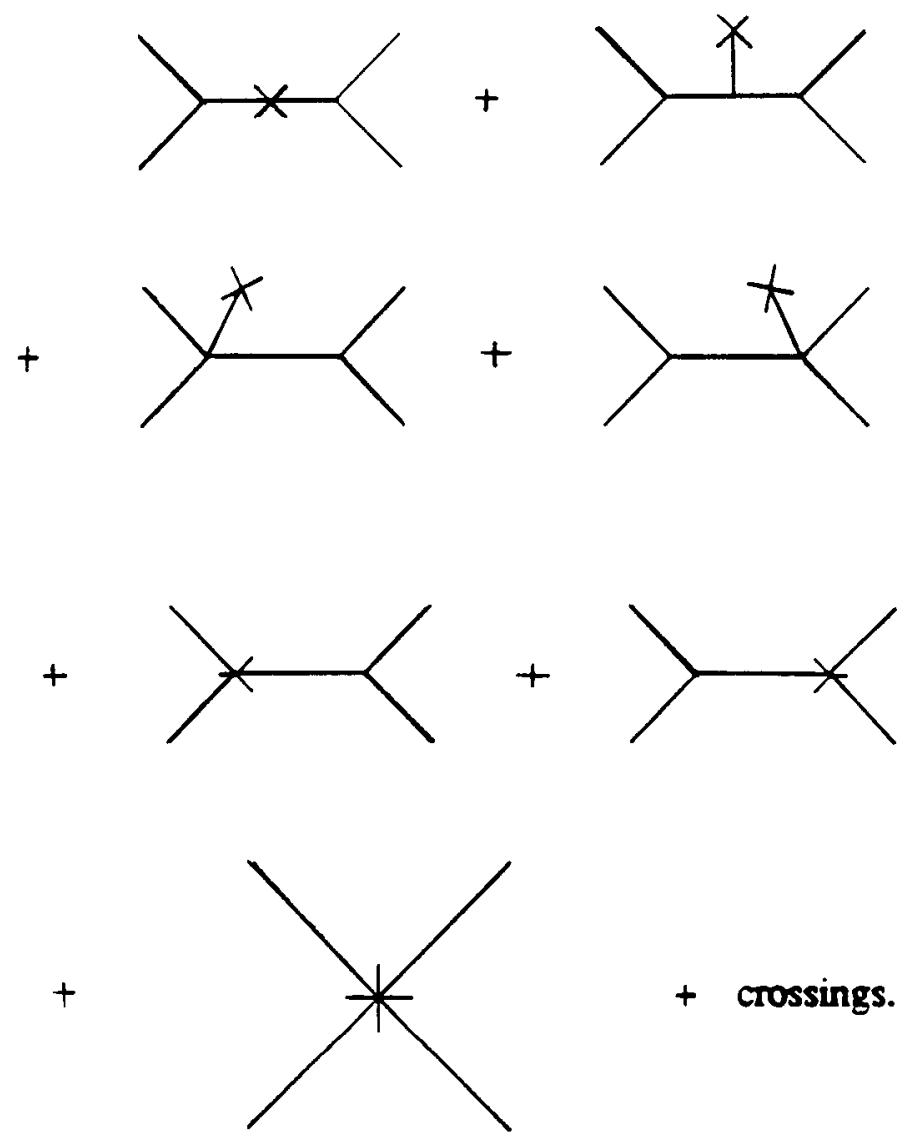

+ crossings.

Figure 2: Counter-terms contribution to $W W$ scattering 
$W_{\mu}^{+} \longrightarrow W_{\nu}^{-} \quad-M^{2}\left(2 \delta_{c}+2 \delta_{M}+\delta_{t}\right)+2 \delta_{c}\left(p . q \delta_{\mu \nu}-p_{\mu} q_{\nu}\right)$

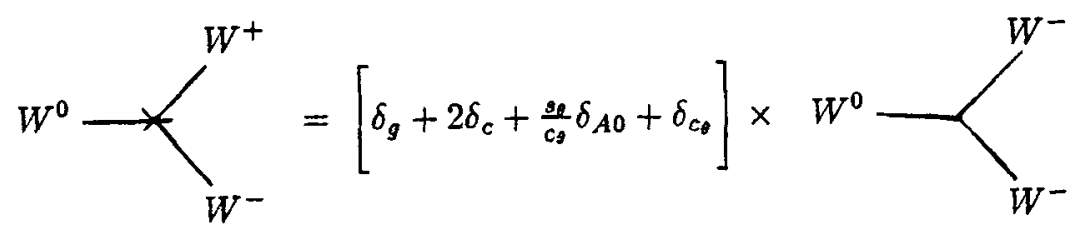<smiles>NC(N)(Br)Br</smiles>

$=\left[2 \delta_{g}+2 \delta_{c}+2 \delta_{0}+2 \frac{s_{\theta}}{c_{\theta}} \delta_{A 0}+2 \delta_{c_{\theta}}\right] \times$<smiles>[Y17]C([17OH])([17OH])[AlH2]</smiles>

Figure 3: Examples of Feynman rules 
Higgs mass $=1,2$ and $10 \mathrm{TeV}$

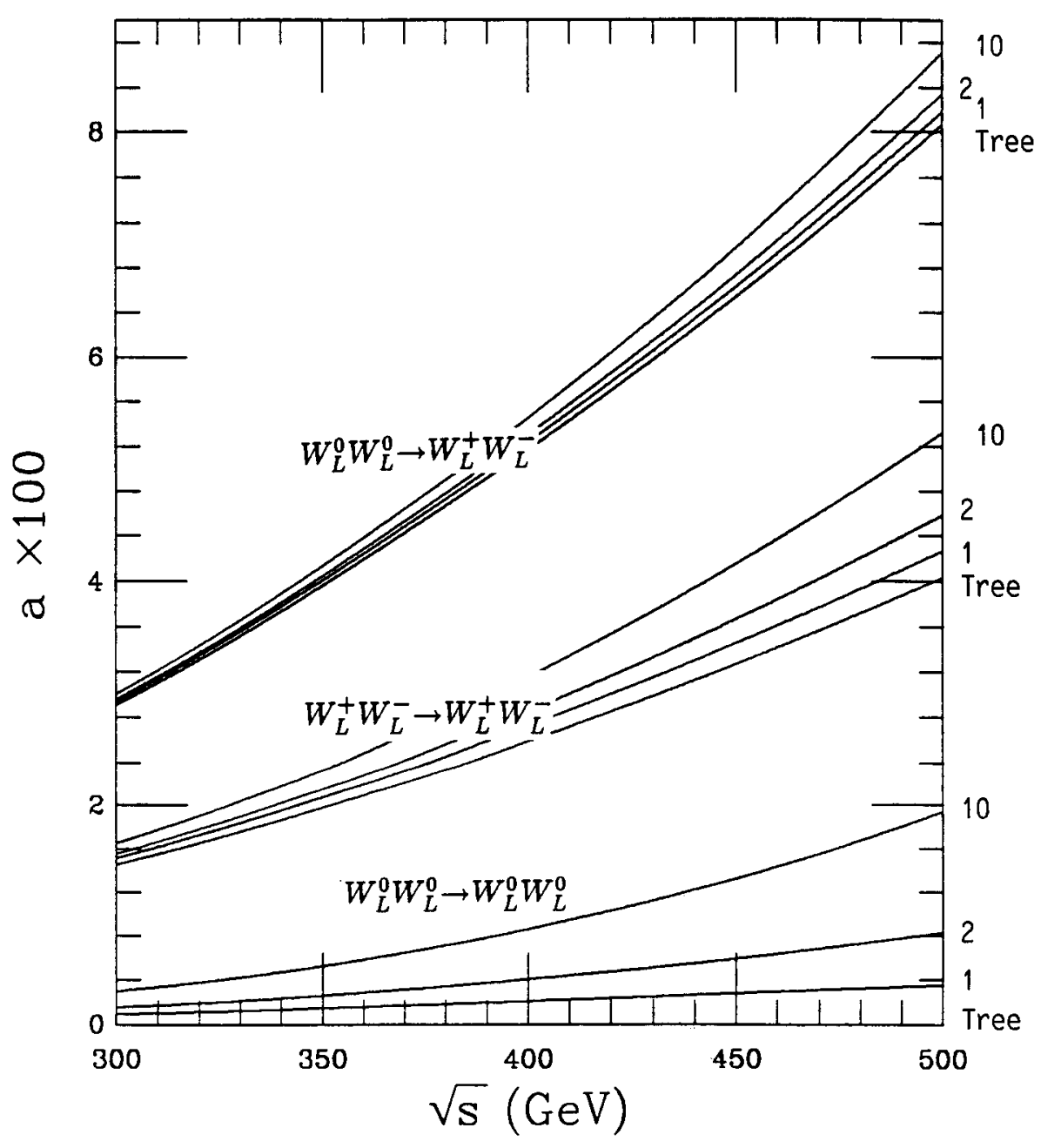

Figure 4: $\mathrm{J}=0$ partial-wave amplitude in the energy window 300 to $500 \mathrm{GeV}$. 


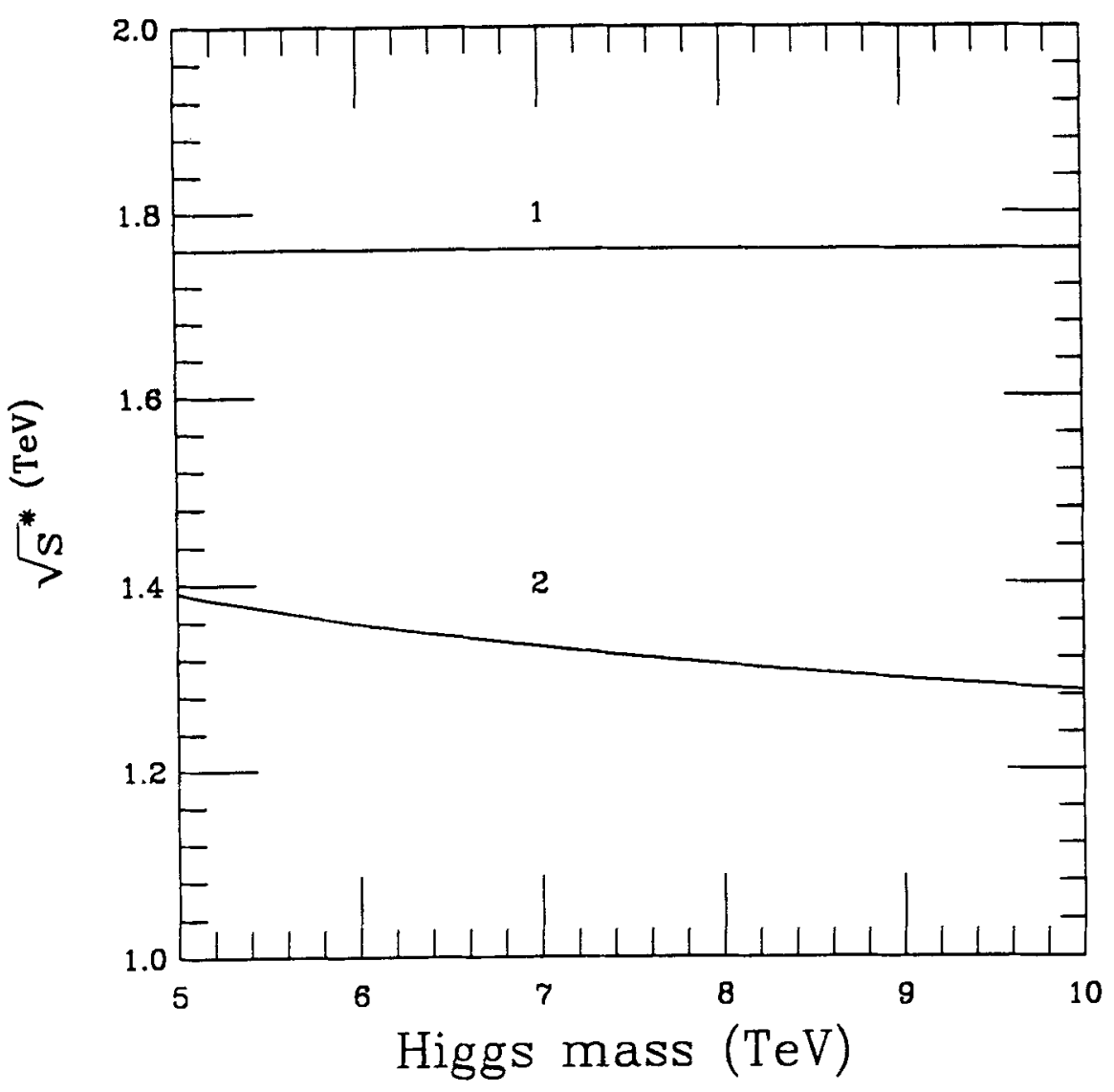

Figure 5: Critical energy as a function of the Higgs mass. Tree level (1). Tree level + one-loop corrections (2). 